\title{
Search for Cluster Emission and Extremely Deformed Shapes using Charged Particle Spectroscopy
}

C. Beck ${ }^{a}$, M. Rousseau ${ }^{a}$, P. Papka ${ }^{a}$, A. Sànchez i Zafra ${ }^{a}$, C. Bhattacharya $^{a, h}$, V. Rauch ${ }^{a}$, P. Bednarczyk ${ }^{a}$, S. Courtin ${ }^{a}$, O.

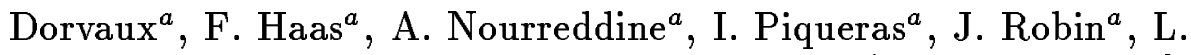
El Bakali ${ }^{a}$, C. Enaux ${ }^{a}$, E. Galmar ${ }^{a}$, S. Szilner ${ }^{a, i}$, O. Stezowski ${ }^{a, b}$, A. Prévost ${ }^{b}$, S. Thummerer ${ }^{c}$, W. von Oertzen ${ }^{c}$, A. Hachem ${ }^{d}$, E. Martin $^{d}$, A. Fahlie, A. Morsad ${ }^{e}$, A. Szanto de Toledo ${ }^{f}$, and S. J. Sanders ${ }^{g}$.

${ }^{a}$ Institut de Recherches Subatomiques, F-67037 Strasbourg, Cedex 2, France

${ }^{b}$ Institut de Physique Nucléaire de Lyon, F-69622 Villeurbanne, France

${ }^{c}$ Hahn-Meitner-Institut Berlin, D-14109 Berlin, Germany

${ }^{d}$ Université de Nice-Sophia-Antipolis, F-06108 Nice, France

${ }^{e}$ Université Hassan II - Mohammadia, Casablanca, Marocco

${ }^{f}$ Instituto de Fisica da Universidade de São Paulo, São Paulo, Brazil

${ }^{g}$ University of Kansas, Lawrence, Kansas 66045, USA

${ }^{h}$ Variable Energy Cyclotron Centre, 1/AF Bidhan Nagar, Kolkata, India

${ }^{i}$ INFN, Laboratori Nazionali di Legnaro, I-35020 Legnaro, Italy

\begin{abstract}
The possible occurence of highly deformed configurations is investigated in the $\mathrm{N}=\mathrm{Z}$ nuclei ${ }^{40} \mathrm{Ca},{ }^{44} \mathrm{Ti}$, and ${ }^{56} \mathrm{Ni}$ as formed in the ${ }^{28} \mathrm{Si}+{ }^{12} \mathrm{C}$, ${ }^{16} \mathrm{O}+{ }^{28} \mathrm{Si}$, and ${ }^{28} \mathrm{Si}+{ }^{28} \mathrm{Si}$ reactions by using the properties of the emitted light charged-particles (LCP). The energy spectra, in-plane and out-of-plane angular correlations of LCP's are analysed for each of the 3 studied reactions within the framework of the statistical model. Strong deformation effects are deduced from Hauser-Feshbach calculations performed with the Monte Carlo code CACARIZO by using a consistent set of parameters with spin-dependent level densities. The analysis of $\alpha$ particles in coincidence with ${ }^{32} \mathrm{~S}$ fragments emitted in ${ }^{28} \mathrm{Si}+{ }^{12} \mathrm{C}$ shows a strong contribution from a ${ }^{8} \mathrm{Be}$ cluster emission. The binary nature of this cluster emission has been verified in three other reactions involving a ${ }^{12} \mathrm{C}$ target: ${ }^{27} \mathrm{Al}+{ }^{12} \mathrm{C},{ }^{31} \mathrm{P}+{ }^{12} \mathrm{C}$, and ${ }^{32} \mathrm{~S}+{ }^{12} \mathrm{C}$.
\end{abstract}

Keywords: Cluster emission, superdeformation, Hauser-Feshbach calculations PACS: 24.60.Dr, 25.70.-z 


\section{Introduction}

Highly deformed shapes and superdeformed (SD) rotational bands have been recently discovered in the $\mathrm{N}=\mathrm{Z}$ nuclei ${ }^{36} \mathrm{Ar}[1],{ }^{40} \mathrm{Ca}[2],{ }^{48} \mathrm{Cr}[3,4]$ and ${ }^{56} \mathrm{Ni}[5]$. Therefore the $\mathrm{A}_{C N} \approx 30-60$ mass region becomes of particular interest since quasimolecular resonances have also been observed for these $\alpha$-like nuclei, in particular, in the ${ }^{28} \mathrm{Si}+{ }^{28} \mathrm{Si}$ reaction $[6,7]$. Although there is no experimental evidence to link the SD bands with the higher lying rotational bands formed by known quasimolecular resonances, both phenomena are believed to originate from highly deformed configurations of these systems.

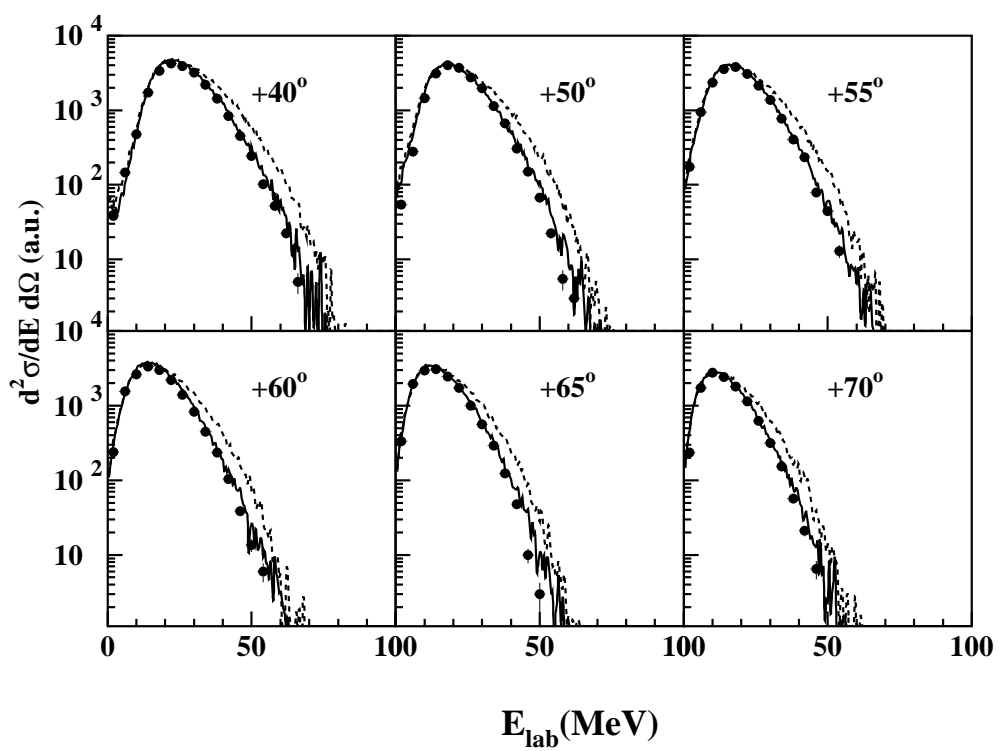

Fig. 1. Exclusive $\alpha$ energy spectra in ${ }^{28} \mathrm{Si}+{ }^{28} \mathrm{Si}$ at $\mathrm{E}_{l a b}=180 \mathrm{MeV}$. Solid and dashed lines are CACARIZO calculations with and without deformations effects.

Since the LCP detection is relatively simple, the analysis of their spectral shapes (see Fig. 1) is another good tool for exploring nuclear deformation and other properties of hot rotating nuclei at high angular momenta [8,9]. The LCP's emitted during the CN decay processes carry information on the underlying nuclear shapes and level densities. In particular, new information on nuclear structure far above the yrast line can be obtained from their study by a comparison with statistical model 
calculations. The properties of the LCP's emitted in the ${ }^{28} \mathrm{Si}+{ }^{28} \mathrm{Si}$ reaction at the bombarding energy $\mathrm{E}_{l a b}=112 \mathrm{MeV}$, which corresponds to the ${ }^{56} \mathrm{Ni}$ excitation energy of the conjectured $\mathrm{J}^{\pi}=38^{+}$quasimolecular resonance $[6,7]$, have already been investigated $[8,10,11]$. The magnitude of the adjustments in the yrast line position suggests deformation effects at high spin for the ${ }^{56} \mathrm{Ni}$ composite system in agreement with very recent $\gamma$-ray spectroscopy data obtained at much lower spins [5]. The extent to which the resonant behaviour is responsible to the observed nuclear deformation is still an open question. To resolve this issue, we have performed a subsequent ${ }^{28} \mathrm{Si}+{ }^{28} \mathrm{Si}$ experiment at $\mathrm{E}_{l a b}=180 \mathrm{MeV}$ outside the "molecular window" where quasimolecular resonances are known to disappear [13]. Deformation effects have also been investigated in ${ }^{28} \mathrm{Si}+{ }^{12} \mathrm{C}$ [9]. The strong cluster emission of ${ }^{8} \mathrm{Be}$ which was observed by this reaction [9] has motivated the search for similar effects in the ${ }^{27} \mathrm{Al}+{ }^{12} \mathrm{C},{ }^{31} \mathrm{P}+{ }^{12} \mathrm{C},{ }^{32} \mathrm{~S}+{ }^{12} \mathrm{C}$, and ${ }^{16} \mathrm{O}+{ }^{28} \mathrm{Si}$ reactions.

\section{The charged particle multidetector array ICARE}

The experiments were performed at the VIVITRON Tandem facility of the IReS Strasbourg laboratory in the ICARE scattering chamber. Both the heavy fragments $(\mathrm{A} \geq 10)$ and their associated LCP's (protons and $\alpha$ particles) were detected in coincidence using the ICARE charged-particle multidetector array $[8,9]$ which consists of nearly 50 telescopes. For the ${ }^{28} \mathrm{Si}+{ }^{28} \mathrm{Si}$ measurement three distinct reaction planes were defined. Two for in-plane correlations and a third one, perpendicular to the LCP detection plane, for out-of-plane correlation measurements. The heavy fragments consisting of evaporation residues (ER) as well as quasi-elastic, deep-inelastic, and fusion-fision fragments, were detected in 8 gas-silicon hybrid telescopes. The in-plane and out-of-plane detection of coincident LCP's was done using 4 three-element telescopes and 16 two-element telescopes with CsI(Tl) scintillators coupled to photodiode readouts. Fig. 2 shows the in-plane LCP angular correlations measured for different ER's $\left(Z=20-23\right.$ ) detected at $\Theta_{\text {lab }}^{E R}=-10^{\circ}$ (see arrows). More details on the experimental setup of ICARE and on the analysis procedures can be found in Refs. [8,9].

\section{Highly deformed ${ }^{56} \mathrm{Ni}$ configurations in ${ }^{28} \mathrm{Si}+{ }^{28} \mathrm{Si}$}

Exclusive $\alpha$ spectra and in-plane angular correlations for both protons and $\alpha$ particles are displayed in Figs. 1 and 2 for ${ }^{28} \mathrm{Si}+{ }^{28} \mathrm{Si}$. The analysis of the data has been performed using CACARIZO [8], the Monte Carlo version of the statistical model code CASCADE. The parameters needed for the statistical description, i.e. the nuclear level densities and the barrier transmission probabilities, are usually obtained from the study of LCP evaporation spectra. The change in the emission barriers and, correspondingly, the transmission probabilities affects the lower energy part of the calculated evaporation spectra. On the other hand the high-energy part of the $\alpha$ spectra depends critically on the available phase space obtained from the 
level densities at high spin. This is clearly shown by the solid and dashed lines of Fig. 1 for ${ }^{28} \mathrm{Si}+{ }^{28} \mathrm{Si}$. The level density, $\rho(E, J)$, for a given angular momentum $J$ and energy $E$ is given by the well known Fermi gas expression:

$$
\rho(E, J)=\frac{(2 J+1)}{12} a^{1 / 2}\left(\frac{\hbar^{2}}{2 \mathcal{J}_{\text {eff }}}\right)^{3 / 2} \frac{1}{\left(E-\Delta-T-E_{J}\right)^{2}} \exp \left(2\left[a\left(E-\Delta-T-E_{J}\right)\right]^{1 / 2}\right)
$$

where $a$ is the level density parameter set equal to $a=\mathrm{A} / 8 \mathrm{MeV}^{-1}$ (A is the mass number), $\mathrm{T}$ is the "nuclear" temperature, and $\Delta$ is the pairing correction, $\mathrm{E}_{J}=$ $\frac{\hbar^{2}}{2 \mathcal{J}_{\text {eff }}} \mathrm{J}(\mathrm{J}+1)$ is the rotational energy, $\mathcal{J}_{\text {eff }}=\mathcal{J}_{\text {sphere }} \times\left(1+\delta_{1} J^{2}+\delta_{2} J^{4}\right)$ is the effective moment of inertia, $\mathcal{J}_{\text {sphere }}=\frac{2}{5} \mathrm{AR}^{2}=\frac{2}{5} \mathrm{~A}^{5 / 3} \mathrm{r}_{0}^{2}$ is the rigid body moment of inertia of a spherical nucleus with radius parameter $\mathrm{r}_{0}$, and $\delta_{1}$ and $\delta_{2}$ are the deformability parameters. The solid lines in Figs. 1 and 2 show the predictions using the same parameter set with deformation effects that has been used at $112 \mathrm{MeV}$ $[8,10,11]$. Predictions with the parameters of the finite-range liquid drop model [14] are unable to reproduce the data (dashed lines). Since the highest incident energy is outside of the "molecular window" [13], we conclude that the highly deformed shapes (with $\beta \approx 0.5$ ) observed for both energies are not related to the ${ }^{28} \mathrm{Si}+{ }^{28} \mathrm{Si}$ quasimolecular resonances $[6,7,13]$.

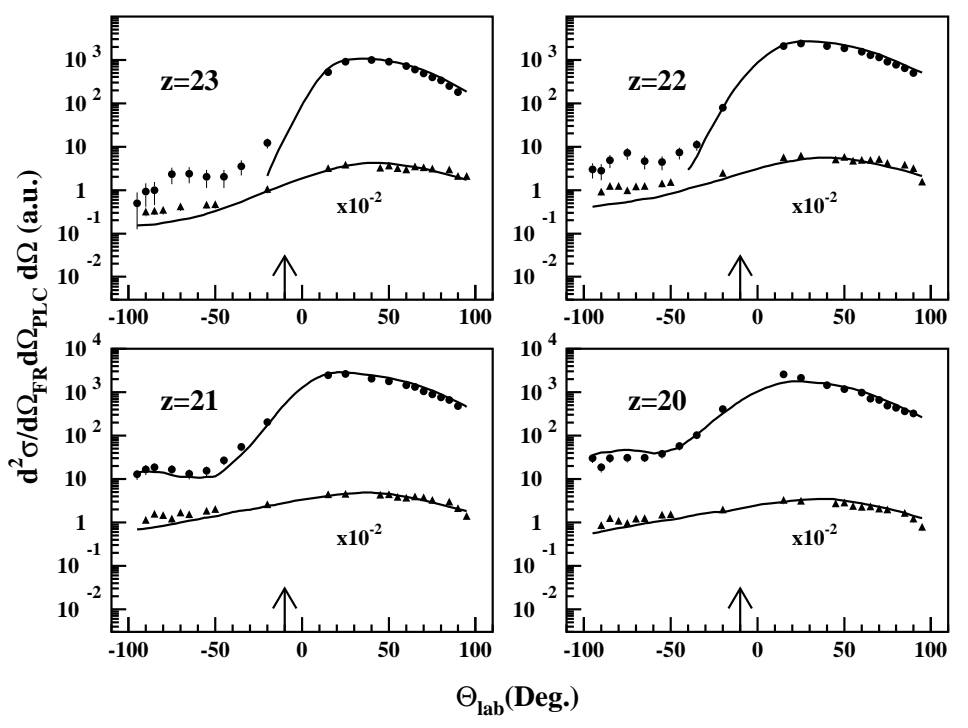

Fig. 2. In-plane angular correlations of $\alpha$ particles (points) and protons (triangles) emitted in coincidence with different ER's $(\mathrm{Z}=20-23)$ in the ${ }^{28} \mathrm{Si}+{ }^{28} \mathrm{Si}$ reaction at $\mathrm{E}_{l a b}=180 \mathrm{MeV}$. The solid lines are CACARIZO calculations with deformation effects discussed in the text (see also Fig. 2). 


\section{Cluster emission versus $\alpha$ evaporation}

Superimposed on the Maxwellian shapes typical of their evaporative origin, nonstatistical $\alpha$-particles components were found in the energy spectra measured in coincidence with $\mathrm{S}$ residues in the ${ }^{28} \mathrm{Si}(180 \mathrm{MeV})+{ }^{12} \mathrm{C}$ reaction [9]. These additional components attributed to the decay of unbound ${ }^{8} \mathrm{Be}$ nuclei are more easily observed in the corresponding energy correlation plot displayed in Fig. 3 (left side of the upper panel). They appear as well defined peaks lying outside the "statistical evaporation region" which is consistent with CACARIZO calculations (see Fig. 10 of Ref. [9]). Their "folding angles" are compatible with the two-body kinematics required for the ${ }^{32} \mathrm{~S}+{ }^{8} \mathrm{Be}$ binary exit-channel. To clearly establish the mechanism resulting in these yields subsequent experiments have been undertaken with ${ }^{12} \mathrm{C}$ targets [15]: ${ }^{27} \mathrm{Al}(150 \mathrm{MeV})+{ }^{12} \mathrm{C},{ }^{31} \mathrm{P}(112 \mathrm{MeV})+{ }^{12} \mathrm{C}$, and ${ }^{32} \mathrm{~S}(140 \mathrm{MeV})+{ }^{12} \mathrm{C}$.
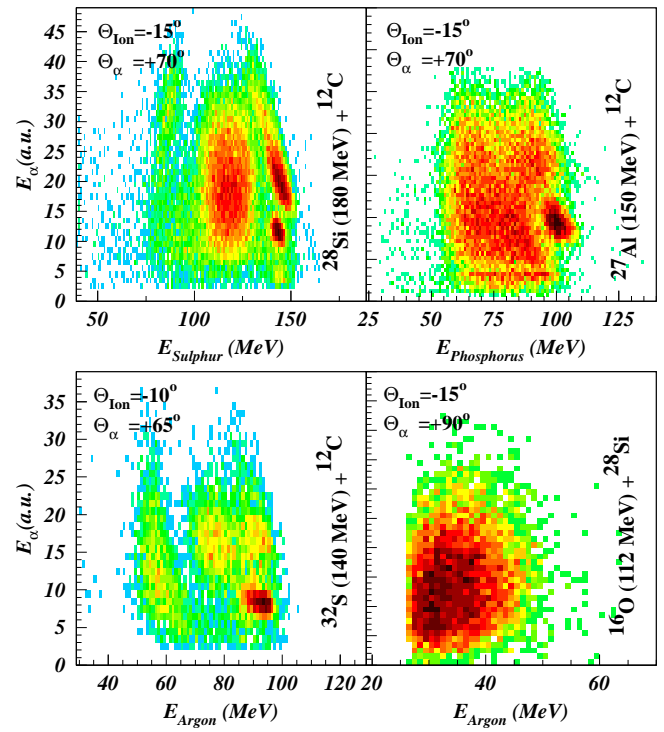

Fig. 3. Energy-correlation plots between $\alpha$ particles and ER's for the ${ }^{28} \mathrm{Si}+{ }^{12} \mathrm{C}$, ${ }^{27} \mathrm{Al}+{ }^{12} \mathrm{C},{ }^{32} \mathrm{~S}+{ }^{12} \mathrm{C}$, and ${ }^{16} \mathrm{O}+{ }^{28} \mathrm{Si}$ reations at the indicated angle settings.

Fig. 3 also displays the energy-correlation plots between $\alpha$ and ER's for two of these reactions [15] and for the ${ }^{16} \mathrm{O}+{ }^{28} \mathrm{Si}$ reaction at $\mathrm{E}_{l a b}\left({ }^{16} \mathrm{O}\right)=112 \mathrm{MeV}[12]$. The fact that the two-body components do not show-up for this last reaction [12] indicates the binary nature of the $\alpha$ peaks present in the reactions involving a ${ }^{12} \mathrm{C}$ target. This hypothesis is consistent with the cluster-transfer picture proposed by Morgenstern et al. [16] for incomplete fusion mechanisms. 


\section{Conclusions}

The occurence of highly deformed configurations in light $\mathrm{N}=\mathrm{Z}$ nuclei has been investigated by using the ICARE charged-particle multidetector array at the VIVITRON Tandem facility of the IReS Strasbourg. The properties of the emitted LCP's in several reactions have been analysed with the CACARIZO statistical-model code that was adapted to calculate evaporation spectra and angular distributions for deformed nuclei. The measured observables such as energy spectra in-plane and out-of-plane angular correlations are well described by Hauser-Feshbach calculations which include spin-dependent level densities. The magnitude of the adjustements in the yrast line suggests deformations at high spins that are far in excess of those predicted by the finite-range liquid drop model [14]. The deformation parameters deduced for ${ }^{28} \mathrm{Si}+{ }^{12} \mathrm{C}$ at $\mathrm{E}_{l a b}\left({ }^{28} \mathrm{Si}\right)=112$ and $180 \mathrm{MeV}[9],{ }^{16} \mathrm{O}+{ }^{28} \mathrm{Si}$ at $\mathrm{E}_{l a b}\left({ }^{16} \mathrm{O}\right)$ $=76,96$, and $112[12]$, and ${ }^{28} \mathrm{Si}+{ }^{28} \mathrm{Si}$ at $\mathrm{E}_{l a b}\left({ }^{28} \mathrm{Si}\right)=112$ and $180 \mathrm{MeV}[8,10,11]$ reactions are comparable to recent $\gamma$-ray spectroscopy data for the ${ }^{40} \mathrm{Ca}$ nucleus [2] and for the ${ }^{56} \mathrm{Ni}$ nucleus [5] at much lower spins. The use of large $\gamma$-ray multidetector arrays will be helpful to extend the existing level scheme of the ${ }^{44} \mathrm{Ti}$ nucleus [17] for the search for weakly populated SD rotational bands equivalent to those discovered in ${ }^{40} \mathrm{Ca}$ [2]. For the ${ }^{28} \mathrm{Si}+{ }^{12} \mathrm{C}$ reaction the component which is found in the $\alpha$-particle energy spectra measured in coincidence with $\mathrm{S}$ residues is attributed to the cluster decay of unbound ${ }^{8} \mathrm{Be}$ nuclei [9]. The hypothesis of the binary nature of the ${ }^{8} \mathrm{Be}$ cluster emission is consistent with the preliminary results found for the three other reactions involving a ${ }^{12} \mathrm{C}$ target [15]: ${ }^{27} \mathrm{Al}+{ }^{12} \mathrm{C}$ at $\mathrm{E}_{l a b}=150 \mathrm{MeV}$, ${ }^{31} \mathrm{P}+{ }^{12} \mathrm{C}$ at $\mathrm{E}_{l a b}=112 \mathrm{MeV}$ and $220 \mathrm{MeV}$, and ${ }^{32} \mathrm{~S}+{ }^{12} \mathrm{C}$ at $\mathrm{E}_{l a b}=140 \mathrm{MeV}$.

\section{References}

1. C.E. Svensson et al., Phys. Rev. Lett. 85 (2000) 2693.

2. E. Ideguchi et al., Phys. Rev. Lett. 87 (2001) 222501.

3. S.M. Lenzi et al., Z. Phys. A354 (1996) 117.

4. S. Thummerer, et al., J. Phys. G: Nucl. Part. Phys. 27 (2001) 1405.

5. D. Rudolph et al., Phys. Rev. Lett. 82(1999) 3763.

6. C. Beck et al., Phys. Rev. C 63 (2001) 014607.

7. R. Nouicer et al., Phys. Rev. C 60 (1999) 41303.

8. C. Bhattacharya et al., Phys. Rev. C 65 (2002) 014611.

9. M. Rousseau et al., Phys. Rev. C 66 (2002) 034612.

10. C. Bhattacharya et al., Nucl. Phys. A654 (1999) 841c.

11. C. Bhattacharya et al., Pramãna J. Phys. 57(2001) 203.

12. P. Papka et al., Acta Phys. Pol. B (2002); Ph.D. thesis (in preparation).

13. C. Beck et al., Phys. Rev. C 49 (1994) 2618.

14. A.J. Sierk, Phys. Rev. C 33 (1986) 2039.

15. A. Sànchez i Zafra, Ph.D. thesis, Strasbourg University (in preparation).

16. H. Morgenstern et al., Z. Phys. A324 (1986) 443.

17. C.D. O'Leary et al., Phys. Rev. C 61 (2000) 064314. 